\title{
ERK-mediated negative feedback regulation of oncogenic EGFRvIII in glioblastoma cells
}

\author{
RATNA DINI HARYUNI ${ }^{1,2}$, TOMOHIRO TANAKA ${ }^{1}$, YUE ZHOU $^{1}$, SATORU YOKOYAMA $^{1}$ and HIROAKI SAKURAI ${ }^{1}$ \\ ${ }^{1}$ Department of Cancer Cell Biology, Faculty of Pharmaceutical Sciences, University of Toyama, \\ Toyama 930-0194, Japan; ${ }^{2}$ Center for Radioisotope and Radiopharmaceutical Technology, \\ National Nuclear Energy Agency of Indonesia, Serpong, Tangerang Selatan 15343, Indonesia
}

Received November 27, 2019; Accepted May 13, 2020

DOI: $10.3892 / \mathrm{ol} .2020 .11760$

\begin{abstract}
Epidermal growth factor receptor variant III (EGFRvIII) is the most common active EGFR mutant in glioblastoma multiforme (GBM). The expression of this mutant often correlates with a poor patient prognosis due to its ability to extend downstream signaling. The EGFR pathway is controlled by a negative feedback mechanism that restricts the extent and length of downstream signaling. To date, the role of negative feedback in the oncogenic EGFRvIII mutant remains undetermined. The present study indicated that activation of the MEK-ERK pathway led to the phosphorylation of Thr-402, a conserved negative feedback residue in the juxtamembrane domain corresponding to Thr-669 of wild-type EGFR (EGFRwt), which resulted in a rapid reduction in the tyrosine phosphorylation of EGFRvIII in U87MG human glioblastoma and 293 cells. Moreover, despite the incapability of EGFRvIII to bind ligands, EGF was indicated to downregulate the tyrosine phosphorylation of EGFRvIII by activating the EGFRwt-ERK pathway. These results demonstrated a conserved negative feedback mechanism in the activation of EGFRvIII, which presents a new aspect in functional interactions between EGFRvIII and EGFRwt in glioblastoma cells.
\end{abstract}

\section{Introduction}

Epidermal growth factor receptor (EGFR), a member of the receptor tyrosine kinase (RTK) family, plays an important role in many fundamental cellular processes, including proliferation, differentiation, migration, and survival (1-3). Due to these crucial functions, an activating mutation in EGFR may lead to malignancies, including non-small cell lung cancer (NSCLC) and glioblastoma multiforme (GBM, glioma grade IV) (4-6).

Correspondence to: Professor Hiroaki Sakurai, Department of Cancer Cell Biology, Faculty of Pharmaceutical Sciences, University of Toyama, 2630 Sugitani, Toyama 930-0194, Japan

E-mail: hsakurai@pha.u-toyama.ac.jp

Key words: epidermal growth factor, epidermal growth factor receptor variant III, ERK, negative feedback, glioblastoma
Although major activating mutations in EGFR occur in the intracellular kinase domain in NLCLCs (7), several deletion mutations in the extracellular domain (ECD) are found in GBM $(1,8)$. An oncogenic variant III (EGFRvIII), which contains a deletion of 267 amino acids spanning exons 2-7 of the EGFR gene, is the most common active mutant and is present in $25-33 \%$ of all GBM patients $(3,9,10)$. This mutation leads to an incapability to bind any known EGFR ligand; however, it exhibits constitutive tyrosine kinase activity (3,11-14). EGFRvIII downstream signaling displays distinct features in signal strength from wild-type EGFR (EGFRwt) $(2,8)$. It has less kinase activity than ligand-activated EGFRwt; however, this constitutive activity is sufficient to provoke downstream signaling (8). EGFRvIII is generally co-expressed with wild-type EGFR (EGFRwt); therefore, the ligand-induced activation of EGFRwt affects the oncogenic potential of EGFRvIII. Discrepancies have been noted in previous findings on the synergistic or antagonistic effects of EGFRwt on EGFRvIII functions (2).

We previously provided evidence for the negative feedback regulation of EGFR/ErbB family kinases by the non-canonical phosphorylation of conserved threonine residues in the juxtamembrane domain (15-17). The ERK kinase, a main regulator of oncogenic EGFR/ErbB signaling, is involved in phosphorylation of the threonine residue, which results in rapid feedback inhibition of the tyrosine kinase activity of ErbB receptor dimers (15).

In the present study, we attempted to investigate the role of the feedback loop in the activation of the EGFRvIII mutant, and found that the ERK-induced phosphorylation of juxtamembrane Thr-402 (corresponding to conserved Thr-669 of EGFRwt) reduced the expression level of constitutively phosphorylated tyrosine in U87MG human glioblastoma cells. We also demonstrated that the EGF-induced activation of EGFRwt rapidly induced the activation of EGFRvIII, and then converted it to an inactivation signal for EGFRvIII via an ERK-mediated feedback mechanism.

\section{Materials and methods}

Antibodies and reagents. Phospho-specific antibodies against EGFR (Tyr-1068 and Thr-669) and ERK (Thr-202/Tyr-204), were purchased from Cell Signaling Technology. Antibodies 
against total EGFR (A-10) and actin (C-11) were obtained from Santa Cruz Biotechnology (Santa Cruz Biotechnology, Inc.). Recombinant human EGF and trametinib were obtained from R\&D Systems and Cayman Chemical, respectively. TPA (12-O-tetradecanoylphorbol-13-acetate) and the Phos-tag ligand were purchased from Wako Pure Chemical Industries. SCH772984 was purchased from Chemietek. All chemical inhibitors were dissolved in dimethyl sulfoxide (DMSO), and the final concentration of DMSO was less than $0.1 \%$.

Cell lines and culture conditions. Human U87MG glioblastoma cells that overexpress EGFRwt and EGFRvIII were provided by Professors Webster K. Cavenee (University of California San Diego) and Motoo Nagane (Kyorin University) $(18,19)$. The original U87MG cells (glioblastoma of unknown origin) were obtained from the American Type Culture Collection. 293 cells were obtained from the ATCC. All cells were cultured in Dulbecco's Modified Eagle's medium (DMEM) supplemented with $10 \%$ fetal calf serum, $2 \mathrm{mM}$ glutamine, $100 \mathrm{U} / \mathrm{ml}$ penicillin, and $100 \mu \mathrm{g} / \mathrm{ml}$ streptomycin at $37^{\circ} \mathrm{C}$ in $5 \% \mathrm{CO}_{2}$.

Cell transfection. Human EGFRvIII cDNA was amplified by reverse transcription-PCR and inserted into the pcDNA3.1 vector. Plasmid DNAs were transfected into 293 cells with Lipofectamine 2000 reagent (Thermo Fisher Scientific, Inc.) following the manufacturer's protocol. The substitution of Thr-669 to Ala was generated by site-directed mutagenesis with KOD FX Neo Polymerase (TOYOBO).

Western blotting. Whole cell lysates were prepared in lysis buffer containing $20 \mathrm{mM} \beta$-glycerophosphate, $1 \mathrm{mM}$ dithiothreitol (DTT), $1 \mathrm{mM}$ sodium orthovanadate, $1 \mathrm{mM}$ phenylmethylsulfonyl fluoride (PMSF), $10 \mu \mathrm{g} / \mathrm{ml}$ aprotinin, and $10 \mu \mathrm{g} / \mathrm{ml}$ leupeptin. Each sample was mixed with the same volume of sample buffer [100 mM Tris- $\mathrm{HCl}(\mathrm{pH} .6 .8), 2.0 \%$ SDS, 70 mM DTT, $10 \%$ glycerol, and $0.10 \%$ bromophenol blue] and then heated at $95^{\circ} \mathrm{C}$ for $5 \mathrm{~min}$. Cell lysates were subjected to $6.5-10 \%$ SDS-PAGE and were then transferred to an Immobilon-P transfer membrane (Merck Millipore Ltd.). The membrane was blocked with BlockAce (Dainippon Sumitomo Pharmaceutical Co., Ltd.) and incubated with an appropriate primary antibody at room temperature. The membrane was then incubated with secondary antibodies, either anti-rabbit or anti-mouse conjugated to horseradish peroxidase (DAKO), diluted in PBS containing 0.1\% Tween-20 (Wako Pure Chemical Industries). Signals were detected with an enhanced chemiluminescence (ECL) system (Thermo Fisher Scientific, Inc.). Some antibody reactions were performed in Can Get Signal solution (TOYOBO).

$\mathrm{Zn}^{2+}$ Phos-tag SDS-PAGE. Whole cell lysates were prepared with RIPA buffer as described previously $(20,21)$. Samples were mixed with a half volume of SDS-PAGE sample buffer [195 mM Tris-HCl (pH. 6.8), 30\% glycerol, 15\% 2-mercaptoethanol, $3 \%$ SDS, and $0.10 \%$ bromophenol blue], and then heated at $95^{\circ} \mathrm{C}$ for $5 \mathrm{~min}$. The acrylamide pendant Phos-tag ligand and two equivalents of $\mathrm{ZnCl}_{2}$ were added to the separating gel before polymerization. The running buffer for $\mathrm{Zn}^{2+}$ Phos-tag electrophoresis consisted of $100 \mathrm{mM}$ Tris and $100 \mathrm{mM}$ MOPS containing $0.1 \%$ SDS and $5.0 \mathrm{mM}$ sodium bisulfite.
Furthermore, the gel was washed in solution containing $25 \mathrm{mM}$ Tris, $192 \mathrm{mM}$ glycine, $10 \%$ methanol, and $1.0 \mathrm{mM}$ EDTA for $20 \mathrm{~min}$, then washed using a solution containing $25 \mathrm{mM}$ Tris, $192 \mathrm{mM}$ glycine, and $10 \%$ methanol for $20 \mathrm{~min}$.

\section{Results}

TPA-induced feedback phosphorylation of EGFR via the $M E K-E R K$ pathway. We initially investigated whether feedback regulation occurs in EGFRwt-overexpressing U87MG human glioma cells. A Phos-tag immunoblot analysis detected a shift in EGFR bands by the TPA stimulation, indicating the strong phosphorylation of EGFR (Fig. 1A). In normal immunoblot analyses, ERK activation and Thr-669 phosphorylation (EGFR phosphorylated on Thr-669 is described as pT-EGFR) were up-regulated in a similar time course (Fig. 1B). Conversely, tyrosine phosphorylation on Tyr-1068 (EGFR phosphorylated on Tyr-1068 is described as pY-EGFR) was gradually decreased (Fig. 1B). In addition, trametinib, a MEK inhibitor (22), completely blocked the negative feedback loop, maintaining pY-EGFR, even in the presence of TPA (Fig. 1C). These results confirmed that the MEK-ERK pathway controlled the negative feedback regulation of EGFRwt by juxtamembrane phosphorylation in glioblastoma cells.

Down-regulation of the constitutive tyrosine phosphorylation of EGFRvIII in 293 cells. We performed transient transfection experiments using 293 cells to characterize the feedback regulation of EGFRvIII. Thr-402 of EGFRvIII is the residue corresponding to feedback Thr-669 of EGFRwt; therefore, Thr-402 of EGFRvIII was also indicated as Thr-669 in the present study. Similar to the results obtained using U87MG-wt cells (Fig. 1A), TPA caused a Phos-tag band shift of EGFRvIII (Fig. 2A). The immunoblot analysis demonstrated that constitutive pY-EGFRvIII completely disappeared with TPA, which inversely correlated with the up-regulation of the ERK-mediated Thr-669 phosphorylation of EGFRvIII (Fig. 2B). To elucidate the role of Thr-669 in feedback inhibition, Thr-669 was substituted to alanine (T669A). The lack of Thr-669 resulted in increased pY-EGFRvIII in the absence of TPA. In addition, although ERK was strongly activated, the down-regulation of pY-EGFRvIII was not observed in the EGFRvIII-T669A mutant (Fig. 2C). Moreover, to elucidate the role of Thr-669 in the EGFRwt/EGFRvIII heterodimer, 293 cells were co-transfected with these EGFR plasmids. TPA efficiently induced the phosphorylation of Thr-669 and dephosphorylation of Tyr-1068 in EGFRwt and EGFRvIII, and trametinib completely blocked these feedback reactions (Fig. 2D). Collectively, these results clearly demonstrated that negative feedback occurred either in the EGFRvIII homodimer or EGFRwt/EGFRvIII heterodimer through ERK-mediated Thr-669 phosphorylation.

Negativefeedbackregulation of EGFRvIII in glioblastoma cells. We attempted to provide direct evidence of negative feedback regulation in glioma cells using U87MG cells overexpressing EGFRvIII. Similar to the results obtained using 293 cells (Fig. 2), TPA induced a Phos-tag band shift and negative feedback reactions of EGFRvIII on Tyr-1068 and Thr-669 in U87MG-vIII cells (Fig. 3A and B). Since U87MG-vIII cells 
A

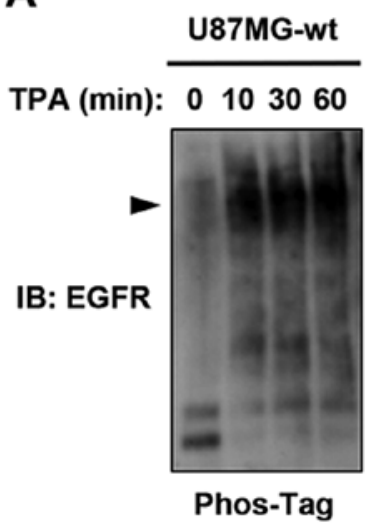

B

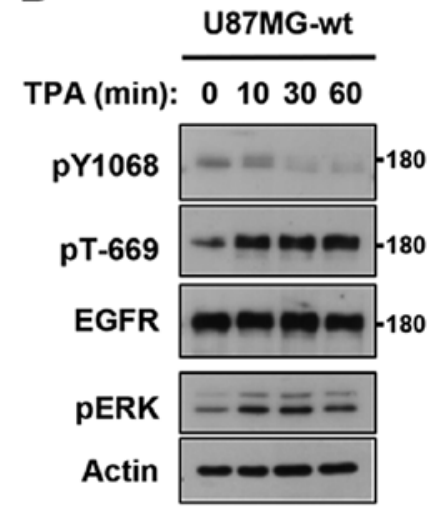

C

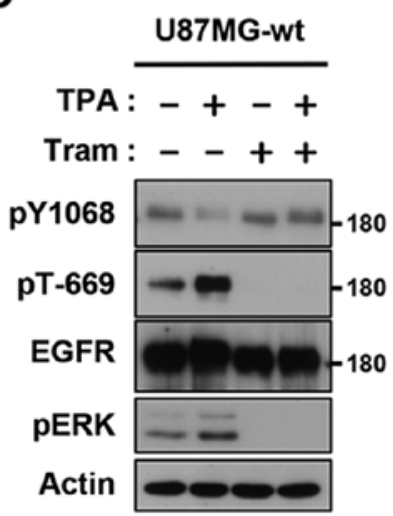

Figure 1. TPA-induced feedback phosphorylation of EGFR via the ERK pathway. (A) Whole cell lysates from U87MG-wt cells stimulated with 100 ng/ml TPA for the indicated periods were separated by $\mathrm{Zn}^{2+}$ Phos-tag SDS-PAGE, followed by immunoblotting (IB) with EGFR antibody. (B) Cells were stimulated with $100 \mathrm{ng} / \mathrm{ml}$ TPA then the whole-cell lysates were electrophoresed and immunoblotted with phospho-EGFR (Thr-669 and Tyr-1068), EGFR, phospho-ERK, and actin antibodies. (C) Cells were pretreated with $0.03 \mu \mathrm{M}$ trametinib (Tram) for 30 min, and then stimulated with TPA for another 10 min. Whole cell lysates were separated by normal SDS-PAGE followed by immunoblotting with primary antibodies against phospho-EGFR (Thr-669 and Tyr-1068), EGFR, phospho-ERK, and actin. EGFR, phospho-ERK and actin antibodies. TPA, 12- $O$-tetradecanoylphorbol-13-acetate; EGFR, epidermal growth factor receptor; wt, wild-type; Tram, trametinib; IB, immunoblotting.

A

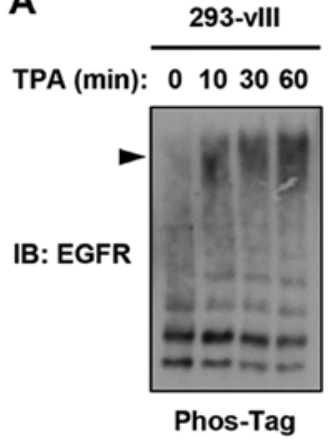

C

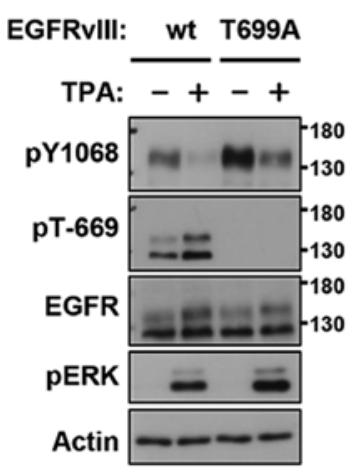

B

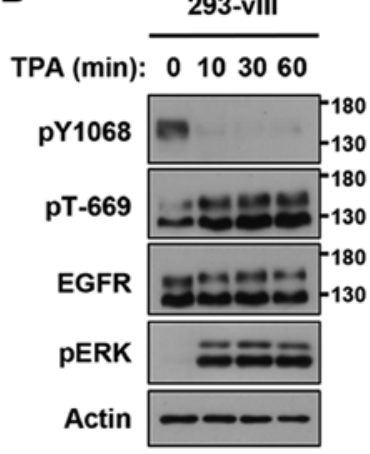

D

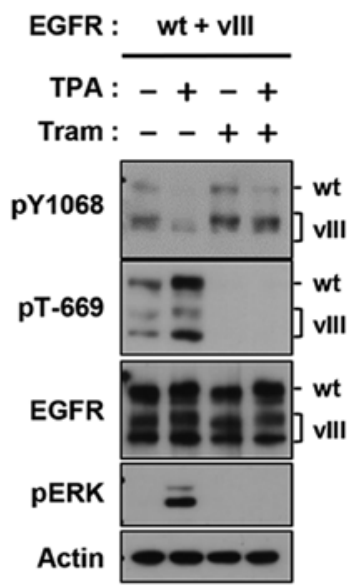

Figure 2. ERK-mediated Thr-669 phosphorylation suppresses the constitutive tyrosine phosphorylation of EGFR-vIII. (A and B) 293 cells transiently transfected with the expression plasmid for EGFR-vIII were stimulated with $100 \mathrm{ng} / \mathrm{ml}$ TPA for the indicated periods. (C) 293 cells transfected with expression plasmids for the WT or T669A mutant of EGFRvIII stimulated with TPA for $10 \mathrm{~min}$. (D) 293 cells co-transfected with EGFRwt and EGFRvIII were pretreated with $0.03 \mu \mathrm{M}$ Tram for $30 \mathrm{~min}$, and then stimulated with TPA for another $10 \mathrm{~min}$. Whole cell lysates were analyzed by (A) $\mathrm{Zn}^{2+}$ Phos-tag-imm unoblotting or (B) normal IB with phospho-EGFR (Thr-669 and Tyr-1068), EGFR, phospho-ERK and actin antibodies. EGFRvIII, epidermal growth factor receptor variant III; TPA, 12- $O$-tetradecanoylphorbol-13-acetate; wt, wild-type; Tram, trametinib; IB, immunoblotting.

constitutively express a basal level of active ERK, we examined the effects of selective inhibitor of MEK1/2, trametinib $(22,23)$, on the phosphorylation of Tyr-1068 and Thr-669. In parallel to the rapid reduction in ERK activation and pT-EGFRvIII, pY-EGFRvIII gradually increased, indicating that basal EGFRvIII activity was also controlled by the phosphorylation of Thr-669 (Fig. 3C). Furthermore, trametinib effectively canceled the TPA-induced feedback regulation of EGFRvIII (Fig. 3D and E). In addition, we investigated the effect of a new selective ERK1/2 inhibitor, SCH772984, on the phosphorylation of Tyr-1068 and Thr-669 (23). This inhibitor also canceled the TPA-induced inhibition of EGFRvIII (Fig. 3E). Overall, these results revealed that ERK activates the Thr-669-dependent EGFRvIII feedback pathway in glioma cells.

Ligand-induced negative feedback inhibition of EGFRvIII. EGFRwt is an important counterpart for EGFRvIII to promote or antagonize EGFRvIII activity (24-26). Since EGFRvIII is frequently co-expressed with EGFRwt in $\operatorname{GBM}(2,6)$, we examined the effects of EGF on the negative feedback regulation of EGFRvIII. The tyrosine phosphorylation of EGFRvIII increased within 1 min, gradually decreased, and was completely suppressed at $60 \mathrm{~min}$ in accordance with the inverse increase in the phosphorylation of ERK and Thr-669 (Fig. 4A). Since EGFRvIII is unable to bind ligands $(3,11,13)$, these inducible events were evoked by endogenous EGFRwt. Thus, the status of EGFRvIII was rapidly changed from the activated to silenced form by the EGF-induced activation of EGFRwt. In addition, the EGF stimulation failed to promote the feedback regulation of EFGRvIII when cells were pretreated with trametinib (Fig. 4B). Collectively, these results demonstrated the physiological feedback inhibition of EGFRvIII as an outcome of ligand stimulation.

\section{Discussion}

Cancer cells may attenuate negative feedback to promote oncogenic growth factor signaling and establish acquired 
A

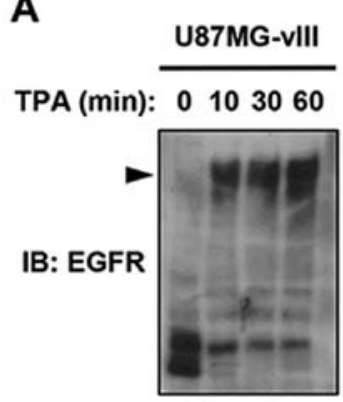

Phos-Tag

D

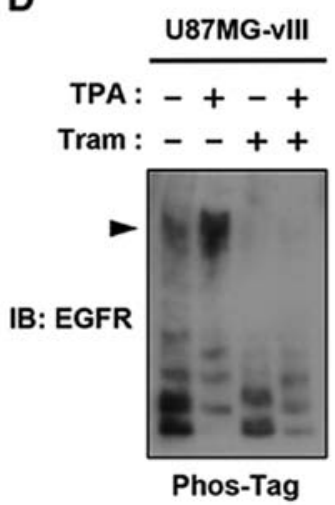

B

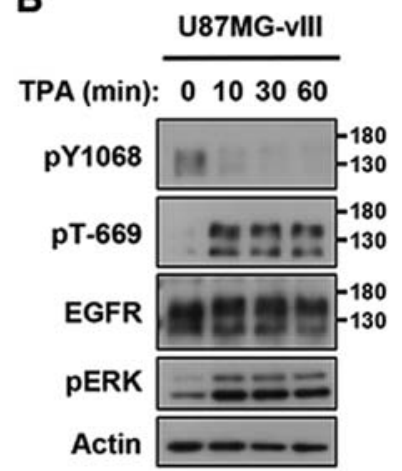

E

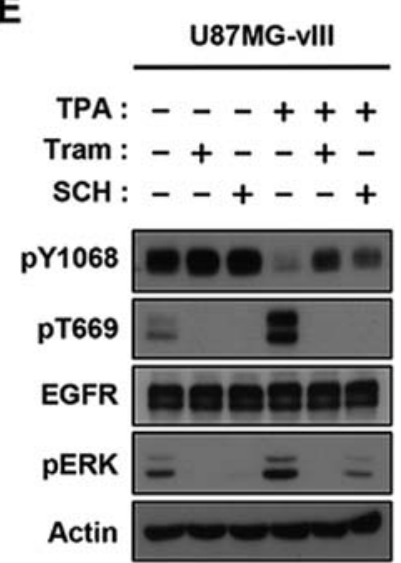

C

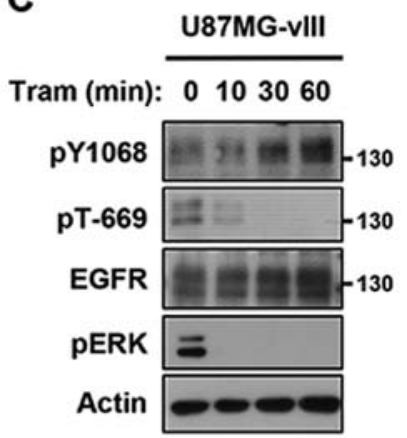

Figure 3. ERK-mediated feedback inhibition of EGFRvIII in glioma cells. (A) U87MG-vIII cells were stimulated with $100 \mathrm{ng} / \mathrm{ml}$ TPA for the indicated periods Whole cell lysates were separated by $\mathrm{Zn}^{2+}$ Phos-tag SDS-PAGE and probed with primary antibody against EGFR. (B) Cells stimulated with $100 \mathrm{ng} / \mathrm{ml} \mathrm{TPA}$ for the indicated periods, then whole cell lysates were analyzed by normal immunoblotting. (C) Cells were treated with $0.03 \mu \mathrm{M}$ trametinib (Tram) for the indicated times, furthermore separated by normal immunoblotting. (D) U87MG-vIII cells were pretreated with $0.03 \mu \mathrm{M}$ trametinib (Tram) $30 \mathrm{~min}$, and then stimulated with TPA for another $10 \mathrm{~min}$. Whole cell lysates were separated by $\mathrm{Zn}^{2+}$ Phos-tag SDS-PAGE and immunoblotted with antibody against EGFR (E) Cells were pretreated with $0.03 \mu \mathrm{M}$ trametinib (Tram) or $0.5 \mu \mathrm{M} \mathrm{SCH772984}$ (SCH) for $30 \mathrm{~min}$, and then stimulated with TPA for another 10 min. All whole cell lysates from normal SDS-PAGE were immnunoblotted with phospho-EGFR (Thr-669 and Tyr-1068). EGFR, phospho-ERK and actin antibodies. EGFRvIII, epidermal growth factor receptor variant III; TPA, 12- $O$-tetradecanoylphorbol-13-acetate; Tram, trametinib; IB, immunoblotting.

A

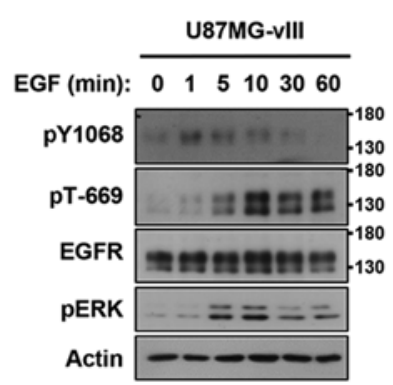

B

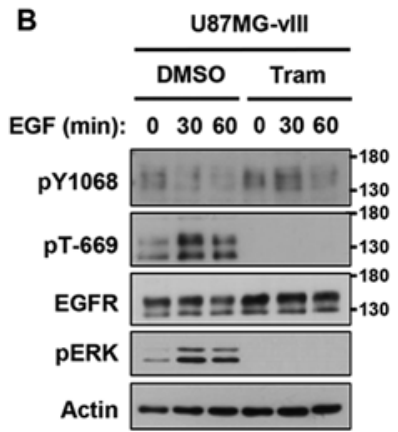

Figure 4. Ligand-induced negative feedback inhibition of EGFRvIII in glioma cells. (A) Cells were stimulated with $10 \mathrm{ng} / \mathrm{ml} \mathrm{EGF}$ for the indicated periods. (B) Cells were pretreated with DMSO or $0.03 \mu \mathrm{M}$ Tram for $30 \mathrm{~min}$, and then stimulated with EGF for the indicated periods. Whole cell lysates were immunoblotted with phospho-EGFR (Thr-669 and Tyr-1068), EGFR, phospho-ERK and actin antibodies. EGFRvIII, epidermal growth factor receptor variant III; EGF, epidermal growth factor; Tram, trametinib.

resistance to anti-cancer agents. We previously reported that the conserved juxtamembrane threonine residue plays a major role in the feedback mechanism of EGFR/ErbB receptor via ERK activation (15-17). Recent study by Soo-Jung Kwon and

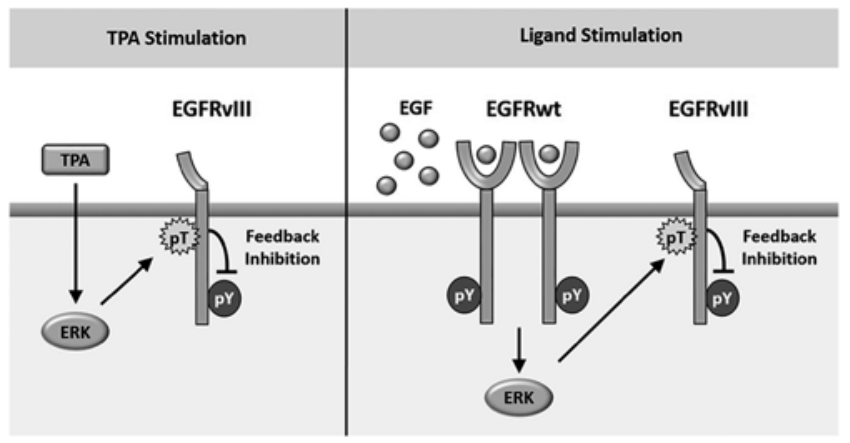

Figure 5. Schematic diagram of negative feedback regulation of EGFRvIII in glioblastoma cells. TPA stimulation leads to down-regulation of the constitutive tyrosine phosphorylation of EGFRvIII via ERK-mediated Thr-669 phosphorylation. Exogenous EGF causes the rapid transactivation of EGFRvIII by EGFRwt, which in turn triggers ERK-mediated feedback inhibition of EGFRvIII. EGFRvIII, epidermal growth factor receptor variant III; TPA, 12-O-tetradecanoylphorbol-13-acetate; wt, wild-type. colleagues demonstrated MEK partner-1 that encoded by LAMTOR3 gene contributes to the neural cancer stemness by control ERK activity in glioma cells expressing EGFRvIII (27). Considering that MEK/ERK pathway is one of important 
downstream signaling in EGFR (28), in the present study we investigated the role of conserved juxtamembrane threonine in EGFRvIII-expressing glioblastoma cells.

There are two opposing observations related to the co-expression of EGFRwt and EGFRvIII in human glioblastoma, namely, synergistic and antagonistic interactions (2). Fan and colleagues reported that EGFR and EGFRvIII collaborated to activate STAT transcription factors (25). In contrast, $\mathrm{Li}$ and colleagues found an antagonistic interaction, in which ligand-induced EGFRwt signaling caused the inactivation of c-Met by inducing the dissociation of a c-Met-EGFRvIII complex (29). A similar antagonistic role was found in the EGFR-mediated activation of NF-kB in glioma cells (30). In order to provide further evidence for this controversial issue, we attempted to elucidate the role of negative feedback phosphorylation in the functional interaction between EGFRwt and EGFRvIII. Although EGFRvIII is incapable of binding ligands, exogenous EGF caused the trans-phosphorylation of EGFRvIII by EGFRwt within one minute (Fig. 4A). More importantly, after the early trans-activation of EGFRvIII, EGF-induced ERK activation caused the feedback suppression of EGFRvIII within $60 \mathrm{~min}$, indicating that the feedback loop is involved in the antagonistic role of EGFRwt in EGFRvIII activation (Fig.5).

The EGFR homodimer, which is composed of an activator and receiver, has been shown to have an asymmetric dimer structure. We previously demonstrated that Thr-669 in only the receiver EGFR was involved in negative feedback. Due to the weak expression of EGFRwt in U87MG-vIII cells, feedback inactivation may occur in EGFRvIII homodimers via EGFRwt-dependent ERK activation. Therefore, the stoichiometric ratio of wild-type and mutant EGFR, which influences the dimer status, will affect the balance between the synergistic and antagonistic activities of EGFRwt signaling in the oncogenic functions of EGFRvIII.

Collectively, these results provide insights into the existence of negative feedback regulation in an oncogenic EGFRvIII deletion mutant, suggesting that the loss of this function contributes to the progression of GBMs. Moreover, MEK inhibition by trametinib counteracted the basal level of the negative feedback regulation of EGFRvIII, resulting in the up-regulation of EGFRvIII activity. Therefore, negative feedback events need to be considered when applying precision medicine to EGFRvIII-expressing glioblastoma.

\section{Acknowledgements}

The authors would like to thank Professor Webster K. Cavenee (University of California San Diego) and Professor Motoo Nagane (Kyorin University) for providing U87MG glioblastoma cells.

\section{Funding}

The present study was supported in part by JSPS KAKENHI (grant nos. 16H04694 and 19H03368) and the Research and Innovation in Science and Technology Project (RISET-Pro) of the Ministry of Research, Technology, and Higher Education of Republic of Indonesia (World Bank Loan no. 8245-ID).

\section{Availability of data and materials}

The datasets used and/or analyzed during the present study are available from the corresponding author on reasonable request.

\section{Authors' contributions}

RDH wrote the manuscript and conducted experiments. TT, YZ and SY designed and helped conduct the experiments. HS designed the study and wrote the manuscript. All authors read and approved the final manuscript.

\section{Ethics approval and consent to participate}

Not applicable.

\section{Patient consent for publication}

Not applicable.

\section{Competing interests}

The authors declare that they have no competing interests.

\section{References}

1. Huang PH, Xu AM and White FM: Oncogenic EGFR signaling networks in glioma. Sci Signal 2: re6, 2009.

2. Guo G, Gong K, Wohlfeld B, Hatanpaa KJ, Zhao D and Habib AA: Ligand-independent EGFR signaling. Cancer Res 75: 3436-3442, 2015

3. Gan HK, Cvrljevic AN and Johns TG: The epidermal growth factor receptor variant III (EGFRvIII): Where wild things are altered. FEBS J 280: 5350-5370, 2013

4. Gazdar AF: Activating and resistance mutations of EGFR in non-small-cell lung cancer: Role in clinical response to EGFR tyrosine kinase inhibitors. Oncogene 28 (Suppl 1): S24-S31, 2009.

5. Taylor TE, Furnari FB and Cavenee WK: Targeting EGFR for treatment of glioblastoma: Molecular basis to overcome resistance. Curr Cancer Drug Targets 12: 197-209, 2012.

6. Padfield E, Hayley P and Kurian KM: Current therapeutic advances targeting EGFR and EGFRvIII in glioblastoma. Front Oncol 5: 5, 2015.

7. Bethune G, Bethune D, Ridgway N and Xu Z: Epidermal growth factor receptor (EGFR) in lung cancer: An overview and update. $\mathrm{J}$ Thorac Dis 2: 48-51, 2010.

8. An Z, Aksoy O, Zheng T, Fan QW and Weiss WA: Epidermal growth factor receptor and EGFRvIII in glioblastoma: Signaling pathways and targeted therapies. Oncogene 37: 1561-1575, 2018.

9. Yang J, Yan J and Liu B: Targeting EGFRvIII for glioblastoma multiforme. Cancer Lett 403: 224-23, 2017.

10. Arteaga CL and Engelman JA: ERBB receptors: From oncogene discovery to basic science to mechanism-based cancer therapeutics. Cancer Cell 25: 282-303, 2014.

11. Gan HK, Kaye AH and Luwor RB: The EGFRvIII variant in glioblastoma multiforme. J Clin Neurosci 16: 748-754, 2009.

12. Tomas A, Futter CE and Eden ER: EGF receptor trafficking: Consequences for signaling and cancer. Trends Cell Biol 24: 26-34, 2014.

13. Chistiakov DA, Chekhonin IV and Chekhonin VP: The EGFR variant III mutant as a target for immunotherapy of glioblastoma multiforme. Eur J Pharmacol 810: 70-82, 2017.

14. Keller S and Schmidt MHH: EGFR and EGFRvIII promote angiogenesis and cell invasion in glioblastoma: Combination therapies for an effective treatment. Int J Mol Sci 18: 1295, 2017.

15. Sato K, Shin MS, Sakimura A, Zhou Y, Tanaka T, Kawanishi M, Kawasaki Y, Yokoyama S, Koizumi K, Saiki I and Sakurai H: Inverse correlation between Thr-669 and constitutive tyrosine phosphorylation in the asymmetric epidermal growth factor receptor dimer conformation. Cancer Sci 104: 1315-1322, 2013. 
16. Kawasaki Y, Sakimura A, Park CM, Tomaru R, Tanaka T, Ozawa T, Zhou Y, Narita K, Kishi H, Muraguchi A and Sakurai H: Feedback control of ErbB2 via ERK-mediated phosphorylation of a conserved threonine in the juxtamembrane domain. Sci Rep 6: 1-9, 2016.

17. Haryuni RD, Watabe S, Yamaguchi A, Fukushi Y, Tanaka T, Kawasaki Y, Zhou Y, Yokoyama S and Sakurai H: Negative feedback regulation of ErbB4 tyrosine kinase activity by ERK-mediated non-canonical phosphorylation. Biochem Biophys Res Commun 514: 456-461, 2019.

18. Nishikawa R, Ji XD, Harmon RC, Lazar CS, Gill GN, Cavenee WK and Huang HJ: A mutant epidermal growth factor receptor common in human glioma confers enhanced tumorigenicity. Proc Natl Acad Sci USA 91: 7727-7731, 1994.

19. Nitta Y, Shimizu S, Shishido-Hara Y, Suzuki K, Shiokawa Y and Nagane M: Nimotuzumab enhances temozolomide-induced growth suppression of glioma cells expressing mutant EGFR in vivo. Cancer Med 5: 486-499, 2016.

20. Zhou Y, Yamada N, Tanaka T, Hori T, Yokoyama S, Hayakawa Y, Yano S, Fukuoka J, Koizumi K, Saiki I and Sakurai H: Crucial roles of RSK in cell motility by catalysing serine phosphorylation of EphA2. Nat Commun 6: 7679, 2015.

21. Kinoshita E and Kinoshita-Kikuta E: Improved Phos-tag SDS-PAGE under neutral $\mathrm{pH}$ conditions for advanced protein phosphorylation profiling. Proteomics 11: 319-323, 2011.

22. Lugowska I, Koseła-Paterczyk H, Kozak K and Rutkowski P: Trametinib: A MEK inhibitor for management of metastatic melanoma. Onco Targets Ther 8: 2251-2259, 2015.

23. Najem A, Krayem M, Perdrix A, Kerger J, Awada A, Journe F and Ghanem G: New drug combination strategies in melanoma: Current status and future direction. Anticancer Res 37: 5941-5953, 2017.
24. Li L, Chakraborty S, Yang CR, Hatanpaa KJ, Cipher DJ, Puliyappadamba VT, Rehman A, Jiwani AJ, Mickey B, Madden C, et al: An EGFR wild type-EGFRvIII-HB-EGF feed-forward loop regulates the activation of EGFRvIII. Oncogene 33: 4253-4264, 2014.

25. Fan QW, Cheng C, Gustafson WC, Charron E, Zipper P, Wong RA, Chen J, Lau J, Knobbe-Thomsen C, Weller M, et al: EGFR phosphorylates tumor-derived EGFRvIII driving STAT3/5 and progression in glioblastoma. Cancer Cell 24: 438-449, 2013.

26. Luwor RB, Zhu HJ, Walker F, Vitali AA, Perera RM, Burgess AW, Scott AM and Johns TG: The tumor-specific de2-7 epidermal growth factor receptor (EGFR) promotes cells survival and heterodimerizes with the wild-type EGFR. Oncogene 23: 6095-6104, 2004.

27. Kwon SJ, Kwon OS, Kim KT, Go YH, Yu SI, Lee BH, Miyoshi H, Oh E, Cho SJ and Cha HJ: Role of MEK partner-1 in cancer stemness through MEK/ERK pathway in cancerous neural stem cells, expressing EGFRviii. Mol Cancer 16: 140, 2017.

28. Wee P and Wang Z: Epidermal growth factor receptor cell proliferation. Cancers (Basel) 9: 52, 2017.

29. Li L, Puliyappadamba VT, Chakraborty S, Rehman A, Vemireddy V, Saha D, Souza RF, Hatanpaa KJ, Koduru P, Burma S, et al: EGFR wild type antagonizes EGFRvIII-mediated activation of Met in glioblastoma. Oncogene 34: 129-134, 2015.

30. Puliyappadamba VT, Chakraborty S, Chauncey SS, Li L, Hatanpaa KJ, Mickey B, Noorani S, Shu HK, Burma S, Boothman DA and Habib AA: Opposing effect of EGFRWT on EGFRvIII-mediated NF- $\kappa$ B activation with RIP1 as a cell death switch. Cell Rep 4: 764-775, 2013. 